\title{
A Nurse-Driven Protocol for Foley Catheter Utilization Decreases the Incidence of Traumatic Foley Catheterization
}

\author{
Eric Laborde, MD, ${ }^{1,2}$ Hayden Hill, MD, ${ }^{1,2}$ Thomas E. Dukovac, MD, ${ }^{1}$ Stephen P. Carriere, MD, ${ }^{3}$ \\ Kathleen Lata-Arias, MPH, ${ }^{1}$ Kristi Hebert, MD, ${ }^{4}$ Raunak Patel, MD, ${ }^{5}$ Jessie Gills, MD ${ }^{6}$ \\ ${ }^{1}$ Department of Urology, Ochsner Clinic Foundation, New Orleans, LA ${ }^{2}$ The University of Queensland Faculty of Medicine, Ochsner Clinical \\ School, New Orleans, LA ${ }^{3}$ Crescent City Urology, New Orleans, LA ${ }^{4}$ Georgia Urology, Atlanta, GA ${ }^{5}$ OhioHealth Physician Group, Columbus, \\ $\mathrm{OH}^{6}$ Department of Urology, Louisiana State University Health Sciences Center, New Orleans, LA
}

\begin{abstract}
Background: Traumatic urethral catheterization is a common reason for urologic consultation in hospitalized patients. The purpose of this study was to determine if a protocol designed to decrease Foley catheter use was effective and if implementation of the protocol decreased the incidence of Foley catheter-associated trauma.

Methods: In an effort to decrease catheter use, our institution adopted a nurse-driven Foley catheter protocol in May 2015 that allowed nurses to remove Foley catheters that did not meet criteria. We conducted a retrospective medical records review of patients who had Foley catheter-associated trauma occurring between February 2013 and March 2018 and compiled data concerning Foley catheter use. Using $t$ test statistical analysis, we compared rates of Foley catheter use and Foley catheter-associated trauma before and after protocol implementation.

Results: During the 62-month study period, we documented 83 cases of Foley catheter-associated trauma. Prior to protocol implementation, our institution had mean of 2,903 patient-catheterization days per month. Following protocol implementation, the mean decreased to 2,604 patient-catheterization days per month $(P<0.01)$. Prior to protocol implementation, the mean incidence of Foley catheter-associated trauma was 1.81 traumas per month. Following protocol implementation, the mean incidence decreased to 0.97 trauma per month $(P<0.05)$.

Conclusion: Implementation of the protocol was successful in decreasing Foley catheter use as well as Foley catheter-associated trauma.
\end{abstract}

Keywords: Catheter-related infections, hematuria, urethral catheterization, urinary catheters, urinary retention, urology

Address correspondence to Eric Laborde, MD, Department of Urology, Ochsner Clinic Foundation, 1514 Jefferson Hwy., New Orleans, LA 70121. Tel: (504) 842-4083. Email: elaborde@ochsner.org

\section{INTRODUCTION}

Traumatic urethral catheterization is a common reason for urologic consultation in hospitalized patients. latrogenic injury associated with urethral catheterization often presents as gross hematuria, severe pain in the penis or perineum, or lack of urine output via the Foley catheter. Such trauma can be a source of significant morbidity. Rarer complications include bladder rupture, perineal and bulbar artery pseudoaneurysm, intraperitoneal catheter misplacement via the verumontanum, autonomic dysreflexia in paralyzed patients, systemic infections, urethrovaginal fistula, and simultaneous bladder and small bowel perforation. ${ }^{1-10}$

Because of the focus on the risk of catheter-associated urinary tract infections (CAUTI) and on methods to reduce the incidence of this complication, ${ }^{11-13}$ many hospitals have implemented protocols to limit Foley catheterization days with the goal of decreasing CAUTI rates. ${ }^{14,15}$

In May 2015, our institution instituted a nurse-driven protocol for Foley removal (Appendix). In the protocol, approved indications for Foley catheterization included urinary retention, nonhealing sacral or perineal wound, required immobilization, hospice/comfort care, critically ill patient in the intensive care unit (ICU) requiring intensive monitoring, chronic indwelling Foley catheter on admission, or any Foley catheter placed or ordered by the urology service. These criteria limit the number of Foley catheters that can be placed. Per the protocol, nurses round daily and have a standing order to remove Foley catheters that do not meet the protocol criteria. Additionally, all physicians were educated about proper indications for Foley catheters, and Foley catheters could be ordered only if a patient met select criteria. We 
hypothesized that the incidence of traumatic Foley catheterization would decrease with the adoption of this protocol.

\section{METHODS}

The urology service compiled a list of consultations for Foley catheter-associated traumas occurring between February 2013 and March 2018. Foley catheter-associated trauma was defined as urologic consultation for gross hematuria immediately following Foley catheter insertion or removal. After obtaining institutional review board approval, we conducted a retrospective medical records review of patients who had Foley catheter-associated trauma during the study period. Data collected included patient age, date of consultation, interventions required, complications, indication for placement, and use of blood thinning medications. Data were also collected on Foley catheter use for all hospitalized patients during the study period.

We compared rates of Foley catheter-associated trauma prior to protocol implementation to the rates after protocol implementation and assessed the rate of traumas per month. We also assessed the number of patient-catheterized days per month before protocol implementation and compared them to patient-catheterized days per month after protocol implementation using a 2-tailed $t$ test. SPSS software, version 21.0 (IBM Corp) was used for statistical analysis.

\section{RESULTS}

During the 62-month study period, we documented 83 cases of trauma secondary to Foley catheterization. In 68 of $83(82 \%)$ cases, the trauma occurred during catheter insertion, and in 15 of 83 cases (18\%), the trauma occurred when patients removed the catheter with the balloon inflated. Twenty-three patients $(28 \%)$ required procedural or operative intervention for their trauma. Demographically, patient age, race, and use of blood-thinning medications were similar before and after implementation of the protocol.

Prior to protocol implementation, 49 cases of Foley catheter-associated trauma occurred in 27 months, for a mean incidence of 1.81 traumas per month. Following protocol implementation, 34 cases of Foley catheter-associated trauma occurred in 35 months, for a mean incidence of 0.97 trauma per month - a $46 \%$ reduction in incidence $(P<0.05)$.

During the 27 months of the study period prior to protocol implementation, the number of patient-catherization days at our institution was 78,380 days, for a mean of 2,903 patientcatheterization days per month. During the 35 months of the study period following protocol implementation, the number of patient-catherization days at our institution was 91,140 days, for a mean of 2,604 patient-catheterization days per month $(P<0.01)$.

\section{DISCUSSION}

Foley catheter-associated trauma is a source of morbidity for patients and historically has been fairly common, with a study from 2008 citing a rate of 3.2 injuries per 1,000 male patient admissions. ${ }^{16}$ This morbidity is often iatrogenic and can be prevented with proper insertion techniques and by avoiding unnecessary catheterization. Sullivan et al reported that the most common complication of urethral catheterization in their study population was iatrogenic ure- thral injury, accounting for $55 \%$ of catheter complications. ${ }^{17}$ Importantly, Sullivan et al reported that for patients who sustained a complication from urethral catheterization, monitoring urinary output was the most common indication cited for urethral catheterization. Routine urinary output monitoring is no longer an acceptable indication for Foley catheterization at our institution. Therefore, avoiding unnecessary urethral catheterization can prevent a large proportion of complications from catheterization, especially urethral injury.

The added morbidity from such complications can lead to the need for surgery and increased length of stay that can significantly increase hospital costs. Davis et al found that patients who sustained an iatrogenic urethral catheter injury had additional costs of $\$ 10,000$ per hospitalization. ${ }^{18}$ Costs included ICU stay, extended hospitalization, bedside and surgical interventions, catheters, and wires. latrogenic urethral catheterization complication increased length of stay by $9.4 \pm 10$ days (range, 2 to 53 days). Davis et al noted that these costs were likely underestimated, as they did not include costs associated with long-term complications, repeat interventions, and follow-up appointments. These results demonstrate that decreasing Foley catheter-associated trauma can improve patient outcomes and reduce the cost of health care.

After implementation of our protocol, the rate of Foley catheter-associated trauma per month decreased. Foley catheter use similarly decreased following implementation of the protocol, supporting the idea that the decrease in the Foley catheter-associated trauma rate is likely related to the decreased utilization. Utilization also highlights that areas for future improvement could focus on making each urethral catheter insertion event safer, possibly by improving the training of nurses and house staff or by focusing on decreasing the inherent risk of the catheters.

A limitation of this study is that we relied on consultations to define Foley catheter-associated trauma. Trauma could have occurred without a consult to the urology service, so all occurrences of trauma may not have been captured.

\section{CONCLUSION}

A protocol designed to decrease Foley catheterization not only significantly decreased the mean number of patientcatherization days per month, but also significantly reduced Foley catheter-associated trauma. We encourage other hospitals to adopt similar protocols.

\section{ACKNOWLEDGMENTS}

The authors have no financial or proprietary interest in the subject matter of this article.

\section{REFERENCES}

1. Poola S, Mohan A. A Foley fallacy: a case of bladder rupture after "routine" Foley catheter placement. Case Rep Urol. 2018;2018:7978126. doi: 10.1155/2018/7978126

2. Oka M, Tomoziri R, Nakashima K. Perforation of the urinary bladder induced by indwelling Foley catheter. Eur Urol. 1980;6(1):53-54. doi: 10.1159/000473289

3. Liang LM, Xue J, Erturk E. Perineal pseudoaneurysm from traumatic Foley removal leads to recurrent life-threatening hematuria. J Endourol Case Rep. 2015;1(1):50-51. doi: $10.1089 /$ cren.2015.0009 
4. Bettez M, Aubé M, Sherbiny ME, Cabrera T, Jednak R. A bulbar artery pseudoaneurysm following traumatic urethral catheterization. Can Urol Assoc J. 2017;11(1-2):E47-E49. doi: 10.5489/cuaj.4050

5. Raheem OA, Jeong YB. Intraperitoneally placed Foley catheter via verumontanum initially presenting as a bladder rupture. $J$ Korean Med Sci. 2011;26(9):1241-1243. doi: $10.3346 / \mathrm{jkms} .2011 .26 .9 .1241$

6. Dave C, Faraj K, Vakharia P, Boura J, Hollander J. Quality improvement Foley project to reduce catheter-related trauma in a large community hospital. J Healthc Qual. 2018;40(1):51-57. doi: 10.1097/JHQ.0000000000000108

7. Vaidyanathan S, Soni BM, Oo T, Hughes PL, Singh G. Missed signs of autonomic dysreflexia in a tetraplegic patient after incorrect placement of urethral Foley catheter: a case report. Patient Saf Surg. 2014;8:44. doi: 10.1186/s13037-014-0044-3

8. Vaidyanathan S, Soni B, Hughes $P$, et al. Candida albicans fungaemia following traumatic urethral catheterisation in a paraplegic patient with diabetes mellitus and candiduria treated by caspofungin. Case Rep Infect Dis. 2013;2013:693480. doi: $10.1155 / 2013 / 693480$

9. Dakhil LOAL. Urethrovaginal fistula: a rare complication of transurethral catheterization. Female Pelvic Med Reconstr Surg. 2014;20(5):293-294. doi: 10.1097/SPV.0000000000000054

10. Amend G, Morganstern BA, Salami SS, Moreira DM, Yaskiv O, Elsamra S. Acute bladder and small bowel perforation as a complication of Foley catheterization. Urology. 2014;83(3):e5-e6. doi: 10.1016/j.urology.2013.11.022

11. Mody L, Greene MT, Meddings J, et al. A national implementation project to prevent catheter-associated urinary tract infection in nursing home residents. JAMA Intern Med. 2017;177(8):1154-1162. doi: 10.1001/jamainternmed.2017.1689
12. Li F, Song M, Xu L, Deng B, Zhu S, Li X. Risk factors for catheter-associated urinary tract infection among hospitalized patients: A systematic review and meta-analysis of observational studies. J Adv Nurs. 2019;75(3):517-527. doi: 10.1111/jan.13863

13. Han CS, Kim S, Radadia KD, et al. Comparison of urinary tract infection rates associated with transurethral catheterization, suprapubic tube and clean intermittent catheterization in the postoperative setting: a network meta-analysis. J Urol. 2017;198(6):1353-1358. doi: 10.1016/j.juro.2017.07.069

14. Hirasaki F, Filho RS, Falci R, et al. Mp70-19 does early withdrawal of urinary catheter following kidney transplant reduce urinary tract infections (UTI)? J Urol. 2019;201(4). doi: 10.1097/01.ju.0000557111.46608.d5

15. Lai CC, Lee CM, Chiang HT, et al. Implementation of a national bundle care program to reduce catheter-associated urinary tract infection in high-risk units of hospitals in Taiwan. $J$ Microbiol Immunol Infect. 2017;50(4):464-470. doi: 10.1016/j.jmii.2017.01.006

16. Kashefi C, Messer K, Barden R, Sexton C, Parsons JK. Incidence and prevention of iatrogenic urethral injuries. J Urol. 2008;179(6):2254-2257; discussion 2257-2258. doi: 10.1016/j.juro.2008.01.108

17. Sullivan JF, Forde JC, Thomas AZ, Creagh TA. Avoidable iatrogenic complications of male urethral catheterisation and inadequate intern training: a 4-year follow-up post implementation of an intern training programme. Surgeon. 2015;13(1):15-18. doi: 10.1016/j.surge.2014.02.001

18. Davis NF, Quinlan MR, Bhatt NR, et al. Incidence, cost, complications and clinical outcomes of iatrogenic urethral catheterization injuries: a prospective multi-institutional study. J Urol. 2016;196(5):1473-1477. doi: 10.1016/j.juro.2016.05.114

This article meets the Accreditation Council for Graduate Medical Education and the American Board of Medical Specialties Maintenance of Certification competencies for Patient Care, Medical Knowledge, and Practice-Based Learning and Improvement.

(C2021 by the author(s); licensee Ochsner Journal, Ochsner Clinic Foundation, New Orleans, LA. This article is an open (c) (i) access article distributed under the terms and conditions of the Creative Commons Attribution (CC BY) license (creativecommons.org/licenses/by/4.0/legalcode) that permits unrestricted use, distribution, and reproduction in any medium, provided the original author(s) and source are credited. 


\section{Appendix}

\section{Operational Standard: Catheter-Associated Urinary Tract Infection (CAUTI) Prevention}

\section{SCOPE:}

This policy applies to Ochsner Medical Center New Orleans (OMC-NO), its employees, medical staff, contract staff regardless of service location, who provide treatment for patients who require an indwelling urinary catheter.

\section{PURPOSE:}

Many hospitalized patients require the placement of temporary indwelling urinary catheters. These patients are at risk for development of catheter-associated urinary tract infections (CAUTI) and complications which increases morbidity, mortality and healthcare costs. OMC-NO utilizes evidence based clinical practice guidelines to reduce the incidence of CAUTI.

\section{POLICY STATEMENT:}

The purpose of this policy is to reduce the incidence of CAUTI by defining care guidelines for all patients who have an indwelling catheter.

\section{DEFINITIONS:}

I. Catheter-associated Urinary Tract Infection (CAUTI): A symptomatic urinary tract infection (UTI) related to an indwelling urinary catheter. The National Healthcare Safety Network (NHSN) definition for CAUTI includes an indwelling catheter in place for $>2$ days with a positive urine culture and at least one sign or symptom of UTI, with specific criteria and time frames for each element (2017).

II. Asymptomatic Bacteriuria (ABU): The presence of one or more bacterial species in the urine without symptoms compatible with UTI (Hooton et al., 2010). Neither screening for nor treatment of $A B U$ is recommended, except in pregnant women, prior to transurethral resection of the prostate, and prior to urologic procedures for which mucosal bleeding is anticipated. Signs and symptoms that should NOT be used to distinguish CAUTI from ABU include pyuria, foul smelling urine, change in urine color, sediment in urine, systemic leukocytosis, or type or number of organisms in the urine (Nicolle et al., 2005).

III. Pyuria: The presence of increased polymorphic leukocytes in the urine, indicative of an inflammatory response in the urinary tract. Pyuria is common in people with $A B U$, and occurs in other infectious and non-infectious conditions. In the catheterized patient, pyuria is diagnostic of neither CAUTI nor ABU, and the presence of pyuria should not be used to distinguish $A B U$ from CAUTI (Hooton et al., 2010; Nicolle et al., 2005).

I. Biofilm: The predominant form of life for the majority of micro-organisms in a hydrated biologic system, biofilm confers survival benefits on many bacterial species. Biofilm consists of adherent microorganisms, their extracellular products, and host components deposited onto the foreign body (Trautner \& Darouiche, 2010; Verma, Bhani, Tomar, Bachhiwal, \& Yadav, 2016). Biofilm development is inevitable with prolonged catheterization, and monthly urine cultures of patients with long-term IUC show constant shifting \& changing of bacterial flora, regardless of antibiotic use (Trautner \& Darouiche, 2010).

IV. Urinary Catheter (UC): A drainage tube made of silicone, latex, or other material that is inserted into the urinary bladder through the urethra to drain urine (Gould et al., 2009). 
V. Indwelling UC (IUC): A UC that is left in place and connected to a closed collection system (Gould et al., 2009). NHSN does not consider suprapubic catheters to be IUCs (2017).

VI. Intermittent Catheterization (IC): Brief insertion of a catheter into the bladder through the urethra to drain urine at intervals. The catheter is immediately removed (Gould et al., 2009).

VII. External catheter: A urine containment device that fits over or adheres to the genitalia and is attached to a urinary drainage bag. The most commonly used external catheter is a soft flexible sheath that fits over the penis ("condom" catheter) (Gould et al., 2009).

\section{GENERAL INFORMATION:}

I. The prevention of catheter-associated urinary tract infection (CAUTI) is a national quality initiative to promote patient safety. Healthcare facilities are challenged to implement evidence-based practices to eliminate CAUTI by reducing indwelling urinary catheter (IUC) utilization, as this is the primary intervention for preventing CAUTI (Lo et al., 2014). As many as $65-70 \%$ of CAUTIs may be preventable (Umscheid et al., 2011).

II. CAUTIs are classified as a "never event" by the Centers for Medicare and Medicaid services and are not reimbursable. CAUTIs are associated with increased morbidity, mortality, length of stay, and exposure to antimicrobials (Gould et al., 2009). The estimated cost of a CAUTI is $\$ 600-1200$ per infection (Zimlichman et al., 2013). IUC use is also associated with other negative outcomes, including urethral inflammation, urethral strictures, mechanical trauma, hematuria, bladder perforation, and mobility impairment (Reilly et al., 2006).

III. Duration of catheterization is the most important risk factor for developing CAUTI. The risk of acquiring bacteriuria increases 3-7\% per day that the IUC is in place (Lo et al., 2014). Because of this, reducing unnecessary catheterizations and minimizing the duration of IUCs are the primary strategies for CAUTI prevention.

IV. Biofilm develops rapidly on the intra- and extra-luminal surfaces of IUCs, and with the proper environment can ascend to the bladder within 1-3 days (Donlan, 2001; Morgan, Rigby, \& Stickler, 2009). The insertion of an IUC initiates an immune response, releasing fibrinogen which rapidly binds to the surface of the IUC and provides an attachment surface for biofilm-producing microbes (Flores-Mireles, Pinkner, \& Hultgren, 2014). Many common uropathogens form biofilm, including Proteus mirabilis, E. coli, and Enterococcus faecalis. Biofilm is frequently polymicrobial, and organisms frequently detach and seed the urine with infection, or form biofilm in a new location (Ganderton et al., 1992; Trautner \& Darouiche, 2010). Bacteria found in biofilm are often resistant strains, as it protects bacteria within from host immune responses, as well as antimicrobial therapy. Biofilm-fed infections usually persist until the colonized surface is removed; biofilm pathogens typically persist by tenacious survival, not aggressive virulence (Stewart \& Costerton, 2001).

V. Inappropriate treatment of asymptomatic catheter-associated bacteriuria (CAABU) promotes antimicrobial overuse, resistance, and development of drug-resistant organisms and infections such as C. difficile (Lo et al., 2014; Mullin et al., 2017). In patients with bacteriuria, the probability of developing a symptomatic CAUTI is $24 \%$ (Goolsarran \& Katz, 2002). Neither bacteriuria nor pyuria alone are of much value in determining if the CAABU is the cause of a patient's fever; in most cases it is not the cause of fever (O'Grady et al., 2008). Bacteremia secondary to CAUTI is rare, and CAUTI is not an independent predictor for increased mortality, although it can be a marker for patients with increased mortality (Laupland et al., 2005). 


\section{APPROPRIATE USE OF FOLEY CATHETERS:}

I. An order is required prior to insertion of an IUC.

a. To be considered complete and valid, the order must include the indication for insertion, described in detail below.

b. The ordering provider should order the Foley Panel in Epic.

i. If the Nurse-Driven Foley Removal Protocol is to be used (OHS.NURS.057), all four components of the Foley Panel should be ordered.

ii. If the Nurse-Driven Foley Removal Protocol is not to be used (inserted by Urology, certain surgical procedures, etc.), all components of the Foley Panel except "Foley catheter - discontinue when patient no longer meets criteria" should be ordered.

c. Patients who are transferred to OMC-NO with an IUC already in place should be assessed for necessity. If the IUC meets one of the criteria outlined in this document, the Foley Panel order should be placed, otherwise promptly remove the IUC.

d. To discontinue the IUC order, simply change the status of the order to discontinued.

i. "Nursing Communication" orders to discontinue an IUC should not be used.

II. Minimize IUC use and duration of use in all patients, particularly those at high risk for CAUTI or mortality from catheterization, including women, the elderly, and patients with impaired immunity (Gould et al., 2009).

a. IUCs should not be used a substitute for nursing care of the patient or resident with incontinence or difficulty mobilizing.

III. Alternatives to an IUC should be considered for patients as appropriate (Gould et al., 2009).

a. Consider external (condom) catheters in males without urinary retention from bladder outlet obstruction.

b. Consider external urine collection device (wicks) in females without urinary retention.

c. Consider intermittent catheterization in patients with bladder emptying dysfunction.

d. Consider alternative measures for urinary output for patients who are not critically ill, such as daily weights, weighing pads for episodes of incontinence, laboratory markers of renal function, bladder scanning, and intermittent catheterization.

IV. Acceptable indications for an IUC (Gould et al., 2009; Meddings et al., 2015):

a. Urinary retention: the patient is unable to pass urine because of an enlarged prostate, blood clots or an edematous scrotum/penis or unable to empty the bladder because of neurologic disease / medication effect, or neurogenic bladder.

i. Intermittent catheterization is preferred to IUCs for the management of most types of urinary retention. Patients with urinary retention managed by intermittent catheterization outside the hospital should continue this while hospitalized, unless hourly intake \& output measurements are required.

ii. Intermittent catheterization does not increase rates of urinary tract infection when compared with IUC, and promotes normal bladder function with the potential for return of spontaneous voiding (Feneley, Hopley, \& Wells, 2015; Lau \& Lam, 2004; Meddings et al., 2015; Purvis et al., 2014).

iii. Intermittent catheterization should NOT be used if the patient has structural contraindication per Urology. 
b. Ordered or placed by Urology service: check plan with Urology service. Do not remove until consulting Urology service.

c. Critically ill in Intensive Care Unit and requiring intensive monitoring and alternatives are not feasible.

i. Placement in critical care alone does not justify the use of an indwelling catheter (Meddings et al., 2015; Reilly et al., 2006).

ii. IUCs may be appropriate for critical care patients who:

1. Require hourly measurements of urinary output to direct treatment

2. Are hemodynamically unstable with titration of fluids/vasoactive drips

iii. IUCs should be removed upon transfer to a lower level of care, unless another indication is met.

d. Non healing perineal and sacral wounds in incontinent patients to avoid further deterioration of wound and skin, if alternatives are not feasible.

i. Wounds must be stage 3 or 4 decubitus and a Wound Care consult note must support use of the IUC for wound healing.

e. Required immobilization for trauma or surgery, if alternatives are not feasible.

i. The immobilization must be medically necessary, including an unstable spine, or multiple traumatic injuries such as pelvic or hip fractures prior to surgical stabilization.

f. Hospice/comfort care at end of life for symptom management, if turning is contraindicated and/or patient's status is terminal and alternatives are not feasible.

i. Urine cultures are not indicated for these patients.

g. Chronic indwelling urinary catheter on admission-must clarify reason for use from physician.

i. Patients with chronic indwelling catheters should be evaluated for the use of alternatives, and if the IUC is to remain in place another indication from this list must be met.

ii. If a determination is made that the IUC is necessary, the primary team treating the patient should change the indication for the catheter on the order in Epic.

iii. Note: Nearly $100 \%$ of patients with a chronic IUC (>30 days) develop bacteriuria. For this reason, routine surveillance for $A B U$ is not recommended, \& caution should be used when interpreting results of urine tests (Saint, 2000).

h. Post Operative order specific to the IUC would supersede this protocol. Cases in this class include those done by Urology, Colorectal, Gynecology, and Transplant Surgery.

i. IUCs should be used in operative patients only as necessary, rather than routinely. Appropriate perioperative uses include:

1. Patients undergoing urologic surgery or other surgery on contiguous structures of the genitourinary tract.

2. Anticipated prolonged ( 3 hours or longer) duration of surgery (catheters inserted for this reason should be removed in PACU).

3. Patients anticipated to receive large-volume infusions or diuretics during surgery.

4. Need for intraoperative monitoring of urinary output. 
ii. Catheters inserted for operative patients should be removed as soon as possible, preferably within 24 hours, unless there is an appropriate indication for continued use.

iii. Presence of a thoracic epidural is not an indication for an IUC (Tripepi-Bova et al., 2013).

V. The following are NOT indications for an IUC (Gould et al., 2009; Meddings et al., 2015):

a. As a substitute for nursing care of the patient with incontinence or immobility that is not medically required.

b. As a means of obtaining urine for culture or other diagnostic tests when the patient can voluntarily void, or a specimen may be obtained using straight catheterization.

c. As part of a fall prevention program.

i. IUCs are not effective in preventing falls, and may actually increase the risk for falls by limiting patient mobility (Krein et al., 2013; Saint, Lipsky, \& Goold, 2002).

d. As a means of obtaining accurate output measurements in patients outside critical care.

i. Urine output may be measured in incontinent patients by weighing pads soaked with urine.

ii. Consider using alternative devices, such as condom catheters, external urine collection devices (wicks), urinals, bedpans, bedside commodes, etc.

iii. Consider using straight catheterization \& bladder scanning (individually or together) to monitor urine output $\&$ bladder volume.

iv. Consider using alternative measures of fluid balance, such as daily weights or laboratory values (creatinine, BUN, etc.).

e. Per patient request for patients who are not on hospice or comfort care for end of life.

f. Morbid obesity.

g. Confusion or dementia.

\section{PROPER TECHNIQUES FOR FOLEY CATHETER INSERTION:}

I. Only trained, licensed staff may insert an IUC.

a. Patient care technicians (PCTs) or other non-licensed personnel may NOT insert IUCs.

b. Staff inserting an IUC should have a documented competency on file (Appendix A).

c. Competency should be assessed upon hire and annually thereafter.

II. If at all possible, IUCs should not be inserted during an emergent situation unless absolutely indicated (i.e. acute urinary retention with potential for bladder rupture), so that aseptic technique may be assured in a more controlled environment.

III. Unless otherwise clinically indicated, consider using a 16 or 18 French catheter, consistent with good drainage, to minimize bladder neck and urethral trauma.

a. The risk of urethral trauma from a false passage goes up as the catheter size goes down, particularly with silicone catheters.

IV. Aseptic technique should be used when inserting an IUC (Gould et al., 2009).

a. It is strongly recommended to have a $2^{\text {nd }}$ person present who is also trained to insert IUCs to assist with patient positioning, monitor sterility of the procedure, \& obtain additional supplies if necessary.

b. It is recommended to have an additional insertion tray at bedside in case of accidental contamination of the first insertion tray, especially for female patients. 
c. It is recommended to have an additional pair of sterile gloves at bedside in case of accidental contamination.

V. Procedure for IUC insertion:

a. Perineal care with soap and water should be performed prior to opening the indwelling catheterization tray.

b. Ask the patient if there have been problems with insertion previously. Ask if allergic to iodine.

c. For pediatric patients, refer to the table in Appendix D for suggested catheter size.

d. Perform hand hygiene prior to procedure, don clean (non-sterile) gloves.

e. Place the female patient in the supine position, knees flexed/separated and feet on bed. Place the male patient in supine position with his legs extended and flat on the bed. If patient is unable to assist/cooperate, use an assistant to hold the patient in the proper position.

f. Open the tray, making a sterile field.

g. Place a sterile drape under the patient's hips (make sure absorbent side of drape is up).

h. Use provided castile soap wipes to cleanse patient's periurethral area using downward strokes from anterior to posterior.

i. Discard gloves, perform hand hygiene with provided alcohol hand sanitizer gel.

j. Maintain aseptic technique and don sterile gloves.

k. Position fenestrated drape on patient as appropriate.

I. Use the syringe with the green plunger to deposit lubricant into tray-top for catheter lubrication.

m. Remove top tray and place next to bottom tray, ensuring that sterile field is maintained.

n. Attach the water-filled syringe to the inflation port.

i. Note: Do not pre-test catheter balloon, this can make insertion more difficult.

o. Remove IUC from wrap and lubricate catheter

p. Prepare patient with packet of pre-saturated antiseptic swab sticks:

i. Female Patient: with a downward stroke cleanse the right labia minora and discard the swab. Repeat for left labia minora. Use the last swab stick to cleanse the area between the labia minora.

ii. Male Patient: Cleanse the penis in a circular motion starting at the urethral meatus and work outward. Foreskin should be retracted in the uncircumcised adult male.

iii. See below for patients with allergies to iodine.

q. Proceed with catheterization until:

i. Female Patient: Advance catheter until urine is seen, then advance two to three more inches.

ii. Male Patient: Advance catheter all the way to the hub ("Y" in tubing next to balloon inflation port). Make sure urine returns. If urine does not return, do not inflate the balloon. Return the foreskin to cover glans if applicable.

iii. Never force the catheter. Stop if resistance is met, Notify charge nurse.

iv. If the catheter becomes contaminated during the procedure, discard catheter and any other contaminated supplies. If the catheter is inadvertently inserted in 
the vagina, leave it there as a landmark. Obtain a new sterile catheter and repeat the procedure.

r. Inflate catheter balloon using entire $10 \mathrm{~mL}$ of sterile water provided in the prefilled syringe.

i. Note: Use of less than $10 \mathrm{~mL}$ can result in improperly inflated balloon

s. After inserting the catheter, discard all materials and remove contaminated gloves, perform hand hygiene.

t. Secure the catheter to the patient using the StatLock device.

u. Position collection bag below the level of the bladder and use green sheeting clip to secure drainage tube to sheet, confirm tube is not kinked.

v. Indicate time and date of catheter insertion on provided labels and place designated label on drainage system.

w. Document in Epic LDA date/time of catheterization, type and size of catheter used, amount and color of urine obtained. The number of insertion attempts and name of the clinician inserting the catheter should also be documented.

VI. Patients with allergies to iodine:

a. Verify what exactly the patient is allergic to - typically it is IV contrast, not topical iodine preparations.

b. If no history of allergic reaction to topical iodine, test a small patch of skin on the forearm.

c. If confirmed reaction to topical iodine, chlorhexidine (CHG) $4 \%$ (with alcohol content $4 \%$ or less) may be used prior to IUC insertion instead of iodine.

i. Prepare CHG-saturated sterile gauze prior to donning sterile gloves and proceeding with insertion of IUC.

d. No evidence exists that $\mathrm{CHG}$ on mucous membranes is harmful - this is routinely used as prep in urologic \& gynecologic surgeries with no adverse effects. Irritation from CHG products on mucous membranes has been linked to higher concentrations of $\mathrm{CHG}$ or especially alcohol (ACOG, 2013; Huang et al., 2014).

PROPER TECHNIQUES FOR IUC MAINTENANCE: (Lo et al., 2014; Gould et al., 2009)

I. Hand hygiene before and after manipulation of the catheter, site, or drainage bag.

II. Properly secure catheters to prevent movement and urethral traction.

a. StatLock placement should allow for 1" slack on the catheter.

b. StatLocks should be placed on the top of the thigh (not the inner thigh/groin) to best promote drainage of urine.

c. StatLocks may also be placed on the abdomen for males with chronic indwelling catheters to prevent erosion of the lower meatus.

III. Maintain a sterile closed drainage system

a. Avoid breaking the red seal between the catheter and drainage bag unless clinically indicated.

i. This may be unavoidable if irrigation is needed, or in other special circumstances. If manual irrigation is required, see the section below on "Manual Irrigation of IUCs".

ii. See the section below regarding changing indwelling urethral catheter and urinary drainage bags. 
b. Do not allow drainage bag to touch floor, even when emptying.

c. Use a dedicated, clean container for emptying drainage bag, change container when soiled.

d. The drainage spout should not touch anything while emptying. If contamination of the drainage spout occurs or is suspected, it should be cleansed with an alcohol or chlorhexidine (CHG) prep pad.

e. Note: Contamination of the drainage spout is suspected to be the main source of entry for pathogens into the IUC drainage system (Tambyah, Halvorson, \& Maki , 1999).

IV. Perineal care performed and documented each shift and when soiled.

a. Perineal care with soap $\&$ water is preferred, $\&$ should be performed each shift and immediately after bowel movements (especially if diarrhea is present).

b. Wash the anal area separately and after each bowel movement with a different cloth.

c. Perineal care includes cleaning the actual catheter from the insertion site to approximately 6" away from the body.

d. Female patients: it is important when cleaning to work from pubis to anal area.

e. Retract the foreskin on uncircumcised male adult patient and clean glans, return the foreskin.

f. Document perineal care in Epic on the LDA (Lines, Drains \& Airways) when performed.

V. Maintain unobstructed urine flow.

a. Use green sheeting clip to prevent dependent loops or kinks.

i. Note: Stasis of urine in the system contributes to biofilm development, which provides an environment for bacteria to multiply and potentially re-seed an infection. Biofilm can develop in as little as 4-8 hours (Kumon et al., 2001; Trautner \& Darouiche, 2010).

ii. Note: Dependent loops create an airlock, preventing full drainage of the bladder and increasing risk of infection (Garcia et al., 2007; Kramer et al., 2017).

b. Empty drainage bag regularly - do not allow overfilling (urine backing up into tubing or bag $>2 / 3$ full).

VI. Maintain drainage bag below level of bladder at all times.

a. Empty drainage bag before transporting patient, do not place bag in bed, on abdomen or between the legs during transportation.

i. Both the bedside RN and the transporter should ensure that the drainage bag is emptied prior to transport.

ii. The box "Foley empty bag on departure" should be checked on the Ticket to Ride.

b. Ensure proper bag position while patient is ambulating.

VII. Use fecal containment device when appropriate for fecal incontinence.

a. Drainage bags for urine and stool should be on opposite sides of the bed.

b. Separate collection containers should be used for each device \& labeled.

VIII. Do not flush catheter unless ordered.

a. The specimen collection port should not be used to flush/irrigate catheters, as this is not effective (Weber, Purvis, VanDenBergh, \& Stevens; 2016).

b. See section below titled "Manual Irrigation of IUCs" for more information.

IX. Patient/family education on the indication for an IUC, risk of developing CAUTI \& prevention methods should be performed \& documented each shift. 
a. The CAUTI FAQ sheet linked in Epic under Group Information may be printed \& given to patients/family members as a handout to support education.

\section{URINE SPECIMEN COLLECTION:}

II. Urine should not be sent for culture \& sensitivity (C\&S) unless there is high suspicion that the patient has a urinary tract infection (Lo et al., 2014; Mullin et al., 2017).

a. A urinalysis (UA) should be ordered prior to a culture \& sensitivity (C\&S).

i. C\&S should only be ordered if the UA results show microscopic $W B C>10$

ii. UA results should be interpreted within the clinical context, especially if multiple potential infectious sources are present (Schwartz \& Barone, 2006).

b. Urine C\&S should be carefully considered in patients at end of life and should be in accordance with the goals of care. If the patient is symptomatic from an infection, narrow spectrum empiric antibiotics (preferably oral) are recommended. If the patient is not symptomatic, no antibiotic is recommended.

c. The clinical significance of CAABU is undefined. Approximately $75 \%$ to $90 \%$ of patients with $A B U$ do not develop a systemic inflammatory response or other signs or symptoms to suggest infection. Monitoring and treatment of CAABU is also not an effective prevention measure for CAUTI, as most cases of CAUTI are not preceded by bacteriuria for more than a day. Treatment of $A B U$ has not been shown to be clinically beneficial and is associated with the selection of antimicrobial-resistant organisms. Fewer than $5 \%$ of bacteriuric cases develop bacteremia (Cope et al., 2009; Gould et al., 2009; Gross \& Patel, 2007; Tambyah \& Maki, 2000).

i. Patients should be carefully evaluated for causes of fever other than bacteriuria.

1. Bacteriuria usually represents colonization, is rarely symptomatic, and is rarely the cause of fever or secondary bloodstream infection, even in immunocompromised patients, unless there is urinary tract obstruction, recent urologic manipulation or surgery, or the patient is granulocytopenic (Leis et al., 2014; O'Grady et al., 2008).

2. Early post-operative fever ( $<72 \mathrm{hrs}$ ) is usually non-infectious. Urine studies are not recommended if fever is the only indication ( $\mathrm{O}^{\prime} \mathrm{Grady}$ et al., 2008).

ii. Certain populations may benefit from treatment of $A B U$, including those undergoing renal transplant, pregnant women, patients about to undergo genito-urinary procedures, and possibly women with bacteriuria after removal of a short-term IUC (Gross \& Patel, 2007; Trautner \& Darouiche, 2010).

III. If an IUC has been in place for 48 hours, or if there is high suspicion of contamination (poor perineal care, discolored tubing, catheter feels gritty when rubbed between fingers, closed system compromised, or break in sterility), the catheter should be changed prior to obtaining the urine for culture. This decreases the chance of specimen contamination with biofilm from internal lumen of IUC \& tubing (Trautner \& Darouiche, 2010).

a. Note: In a randomized trial, patients who had their IUC changed prior to obtaining C\&S \& initiating antimicrobial therapy had a decreased duration of fever, improved clinical status after 72 hours of therapy, and a lower rate of symptomatic relapse after 28 days (Raz, Schiller, \& Nicolle, 2000). 
IV. If an external device (condom catheter or wick) is in use, urine for C\&S should be obtained via straight catheterization.

a. Urine for studies other than C\&S may be collected from the external device if the perineum is cleansed and a fresh device, tubing, and bag/canister are used.

V. Procedure for urine specimen collection:

a. Wash hands \& don gloves.

b. Kink catheter tubing approximately 3 " below the specimen port. Wait until urine appears under the specimen port.

c. Cleanse specimen port thoroughly with an alcohol/CHG wipe.

d. Attach syringe to the specimen port and aspirate appropriate urine amount.

e. Place urine in specimen container and label with patient's ID Label, date and time, and whether specimen source is from catheter or clean catch.

f. Remove gloves and wash hands.

g. Transport specimen to the lab within 1 hour.

h. Note: If urine cannot be transported to the lab within 1 hour, the specimen should be refrigerated (O'Grady et al., 2008).

\section{CHANGING INDWELLING URETHRAL CATHETER AND URINARY DRAINAGE BAG:}

I. Routine catheter and drainage system changes at fixed intervals are not recommended (Gould et al., 2009; Lo et al., 2014). Change catheter when clinically indicated (e.g. Urinary Tract Infection [UTI], obstruction, or when closed system is compromised and there is an observed break in sterility).

a. If there is concern for infection risk with unavoidable prolonged catheterization, catheters should be removed and replaced no more frequently than 21 days.

b. See Appendix C for guidance on patients admitted with an IUC.

c. See the previous section with regard to changing catheters prior to obtaining urine specimens.

d. When changing a catheter, if possible, allow the patient to void at least once (even if incontinent) between discontinuing the old IUC \& inserting the new IUC. The natural flushing action of urine helps to clear the urethral tract of residual microbes that may be pushed back into the bladder during immediate reinsertion (Feneley et al., 2015; Stickler, 2014; Trautner \& Darouiche, 2010). Further, immediate replacement of catheters in patients with bacteriuria allows rapid development of biofilm, providing a source for re-infection (Morgan, Rigby, \& Stickler, 2009).

II. Maintenance of a closed system is recommended. Change of urinary drainage bag should be avoided unless needed (e.g., leakage, need for urimeter, etc.).

a. If a change of the bag is indicated and there is no suspicion of contamination (cloudy urine, sediment, discolored tubing, catheter feels gritty when rubbed between fingers), the drainage bag may be changed using aseptic technique.

b. While changing the bag, ensure that the open end of the catheter is not contaminated. Prior to attaching the new drainage bag, cleanse the connection between the indwelling catheter and the drainage tubing with antiseptic swabs.

\section{PROMPT REMOVAL OF IUCS:}


I. Nursing should assess for the presence of an IUC and appropriate indication each shift.

a. The bedside nurse should also assess the patient's suitability for external devices, such as condom catheters for males or wick devices for females. If a patient is able to be managed using an alternative, the IUC should be discontinued.

b. The reason for continuing catheterization should be documented on the LDA (Lines, Drains \& Airways) in Epic each shift.

c. The indication for the IUC that was originally entered on the order may no longer be valid; it is more important that nursing documentation reflect the patient's current clinical condition versus using the same indication that appears on the order.

II. If there is not an appropriate indication for the IUC, the nurse should remove the IUC and discontinue the order in Epic per OHS.NURS.057 - Nurse Driven Protocol for Foley Removal Policy.

III. Clamping indwelling catheters prior to removal is not necessary and may actually increase the risk of developing a CAUTI.

IV. Procedure for removal of an IUC:

a. Explain the procedure to the patient and ensure privacy

b. Perform hand hygiene, don clean non-sterile gloves.

c. Remove StatLock stabilization device.

d. Position patient appropriately and place waterproof pad under patient.

e. Deflate catheter balloon:

i. Back off syringe to $0.5 \mathrm{~mL}$ and insert into the inflation port.

ii. Allow the water to naturally flow back into the syringe to deflate the balloon

iii. Remove all $10 \mathrm{~mL}$ of water.

iv. Do not use vigorous aspiration as this may cause the inflation lumen to collapse, preventing balloon deflation.

v. Use only gentle aspiration to encourage deflation if needed.

vi. Allow approximately 30 seconds for the pressure within the balloon to force the plunger back and volunteer its water into the syringe.

vii. If slow or no deflation is noticed, reseat the syringe.

viii. Note: If passive deflation of catheter balloons is not used, there is a risk of urethral trauma and/or catheter balloon rupture with or without retained catheter fragments. This risk is increased with silicone catheters (Feneley et al., 2015; Parkin et al., 2002).

f. Slowly remove the catheter steadily and smoothly. If resistance is met, check to be sure all fluid was aspirated from balloon.

g. Dispose of all supplies.

h. Remove gloves and perform hand hygiene.

i. After removal, monitor patient for signs of retention including feeling of fullness, cramping, painful urination, difficulty urinating, frequent urination in small amounts. Perform bladder scanning as directed by post-catheter removal algorithm in OHS.NURS.057.

V. Patients who refuse to have an IUC discontinued should be counseled each shift by the bedside $\mathrm{RN}$ as well as the Primary Team regarding the risks of continued catheterization, lack of necessity, $\&$ alternative options.

a. This conversation and the patient's continued refusal should be documented daily by both the nurse and the primary team in Notes.

b. If the patient continues to refuse to have an IUC removed, the bedside RN and/or Primary Team may contact Infection Control for additional support (Appendix B). 


\section{MANUAL IRRIGATION OF IUCS:}

I. See Mosby's Nursing Skills:

II. Bladder irrigation is not recommended unless obstruction is anticipated.

III. Manual bladder irrigation should be performed using aseptic technique with sterile saline and sterile syringe.

IV. Forceful irrigations may be needed for grossly hematuric patients (i.e. transurethral resection of prostate). Irrigations should continue until urine is clear of clots or hematuria.

\section{QUALITY MONITORING:}

I. If a CAUTI occurs, the unit director (UD) or designee for the unit on which the CAUTI occurred will complete the deep dive tool within 1 week of the occurrence.

a. The UD, in collaboration with the Unit Based Medical Director (UBMD) will ensure that providers who cared for the patient over the course of catheterization are aware that the patient developed a CAUTI, \& that they are familiar with the CAUTI prevention plan.

b. The patient's attending physician will also be involved in the investigation into development of the CAUTI and action planning for prevention of future incidents. It will be the responsibility of the UBMD to ensure all responsible providers are included in this process.

c. The UD and UMBD should use the tool and review process to inform unit-specific performance improvement efforts.

II. The deep dive tool will be reviewed and compared with hospital-wide trends by the Infection Preventionist and others as needed to identify opportunities for improvement. 
References

American College of Obstetricians and Gynecologists [ACOG] Women's Health Care Physicians: Committee on Gynecologic Practice. (2013). Solutions for surgical preparation of the vagina. Obstetrics and Gynecology, 122, 718-720.

Cope, M., Cevallos, M. E., Cadle, R. M., Darouiche, R. O., Musher, D. M., \& Trautner, B. W. (2009). Inappropriate treatment of catheter-associated asymptomatic bacteriuria in a tertiary care hospital. Clinical Infectious Diseases, 48, 1182-8.

Donlan, R. M. (2001). Biofilms and device-associated infections. Emerging Infectious Diseases, 7, 277-81.

Feneley, R. C., Hopley, I. B., \& Wells, P. N. T. (2015). Urinary catheters: history, current status, adverse events and research agenda. Journal of Medical Engineering \& Technology, 39, 459-70.

Flores-Mireles, A. L., Pinkner, M. G., \& Hultgren, S. J. (2014). EbpA vaccine antibodies block binding of Enterococcus faecalis to fibrinogen to prevent catheter-associated bladder infection in mice. Science Translational Medicine, 6, Supplement.

Ganderton, L., Chawla, J., Winters, C., Wimpenny, J., \& Stickler, D. (1992). Scanning electron microscopy of bacterial biofilms on indwelling bladder catheters. European Journal of Clinical Microbiology and Infectious Diseases, 11, 789-96.

Garcia, M. M., Gulati, S., Liepmann, D., Stackhouse, G. B., Greene, K., \& Stoller, M. L. (2007). Traditional Foley drainage systems - Do they drain the bladder? The Journal of Urology, 177, 203-7.

Goolsarran, V. J., \& Katz, T. F. (2002). Do not go with the flow, remember indwelling catheters. Journal of the American Geriatrics Society, 50, 1739-40.

Gould, C., Umscheid, C. A., Agarwal, R. K., Kuntz, G., Pegues, D. A., \& Healthcare Infection Control Practices Advisory Committe (HICPAC). (2009). Guideline for prevention of catheter-associated urinary tract infections (CDC Guideline). Retrieved from http://www.cdc.gov/hicpac/ cauti/02_cauti2009_abbrev.html

Gross, P. A., \& Patel, B. (2007). Reducing antibiotic overuse: a call for a national performance measure for not treating asymptomatic bacteriuria. Clinical Infectious Diseases, 48, 1182-8.

Tambyah, P. A., Halvorson, K. T., \& Maki, D. G. (1999). A prospective study of pathogenesis of catheter-associated urinary tract infections. Mayo Clinic Proceedings, 74, 131-6.

Hooton, T. M., Bradley, S. F., Cardenas, D. D., Colgan, R., Geerlings, S. E., . . Nicolle, L. E. (2010). Diagnosis, prevention, and treatment of catheter-associated urinary tract infection in adults: 2009 International Clinical Practice Guidelines from the Infectious Diseases Society of America. Clinical Infectious Diseases, 50, 625-63.

Huang, S. S., Septimus, E., Avery, T. R., Lee, G. M., Hickok, J., Weinstein, R. A., . . Ray, G. T. (2014). Cost Savings of Universal Decolonization to Prevent Intensive Care Unit Infection: Implications of the REDUCE MRSA Trial. Infection Control and Hospital Epidemiology, 35, S23-S31.

Kramer, G. C., Luxon, E., Wolf, J., Burnett, D. R., Nanduri, D., \& Friedman, B. C. (2017). Inaccuracy of urine output measurements due to urinary retention in catheterized patients in the burn ICU. Journal of Burn Care \& Research, 38, e409-17.

Krein, S. L., Kowalski, C. P., Harrod, M., Forman, J., \& Saint, S. (2013). Barriers to reducing urinary catheter use: A qualitative assessment of a statewide initiative. JAMA Internal Medicine, 173, 881-886.

Kumon, H., Hashimoto, H., Nichimura, M., Monden, K., \& Ono, N. (2001). Catheter-associated urinary tract infections: Impact of catheter materials on their management. International Journal of Antimicrobial Agents, 17, 311-6. 
Lau, H. \& Lam, B. (2004). Management of postoperative urinary retention: A randomized controlled trial of in-out versus overnight catheterization. ANZ Journal of Surgery, 74, 658-61.

Laupland, K. B., Bagshaw, S. M., Gregson, D. B., Kirkpatrick, A. W., Ross, T., \& Church, D. L. (2005). Intensive care unit-acquired urinary tract infections in a regional critical care system. Critical Care, 9, R60-5.

Leis, J. A., Rebick, B. W., Daneman, N., Gold, W. L., Poutanen, S. M., . . McGeer, A. (2014). Reducing antimicrobial therapy for asymptomatic bacteriuria among noncatheterized inpatients: A proof-of-concept study. Clinical \& Infectious Diseases, 58, 980-3.

Lo, E., Nicolle, L. E., Coffin, S. E., Gould,C., Maragakis, L. L., . . Yokoe, D. S. (2014). Strategies to prevent catheter-associated urinary tract infections in acute care hospitals: 2014 update. Infection Control \& Hospital Epidemiology, 35, 464-479.

Meddings, J., Saint, S., Fowler, K. E., Gaies, E., Hickner, A., Krein, S. L., \& Bernstein, S. J. (2015). The Ann Arbor Criteria for Appropriate Urinary Catheter Use in Hospitalized Medical Patients: Results Obtained by Using the RAND/UCLA Appropriateness Method. Annals of Internal Medicine, 162, S1-S34.

Morgan, S. D., Rigby, D., \& Stickler, D. J. (2009). A study of the structure of the crystalline bacterial biofilms that can encrust and block silver Foley catheters. Urological Research, 37, 89-93.

Mullin, K. M., Kovacs, C. S., Fatica, C., Einloth, C., Neuner, E. A., . . Fraser, T. G. (2017). A Multifaceted Approach to Reduction of CAUTI in the ICU with an emphasis on "stewardship of culturing." Infection Control \& Hospital Epidemiology, 38, 186-188.

National Healthcare Safety Network (NHSN) patient safety component manual. (2017). Retrieved from https://www.cdc.gov/nhsn

Nicolle, L. E., Bradley, S., Colga, R., Rice, J. C., Schaeffer, A., \& Hooton, T. M. (2005). Infectious Diseases Society of America (IDSA) guidelines for the diagnosis and treatment of asymptomatic bacteriuria in adults. Clinical Infectious Diseases, 40, 643-54.

O'Grady, N. P., Barie, P. S., Bartlett, J. G., Bleck, T., Carroll, K. . . Masur, H. (2008). Guidelines for evaluation of new fever in critically ill adult patients: 2008 update from the American College of Critical Care Medicine and the Infectious Diseases Society of America. Critical Care Medicine, 36, 1330-49.

Parkin, J., Scanlan, J., Woolley, M., Grover, D., Evans, A., \& Feneley, R. C. L. (2002). Urinary catheter 'deflation cuff' formation: Clinical audit and quantitative in vitro analysis. BJU International, 90, 666-71.

Purvis, S., Gion, T., Kennedy, G., Rees, S., Safdar, N., . . Weber, J. (2014). Catheter-associated urinary tract infection: A successful prevention effort employing a multipronged initiative at an academic medical center. Journal of Nursing Care Quality, 29, 141-148.

Raz, R., Schiller, D., \& Nicolle, L. E. (2000). Chronic indwelling catheter replacement before antimicrobial therapy for symptomatic urinary tract infection. The Journal of Urology, 164, 1254-8.

Reilly, L., Sullivan, P., Ninni, S., Fochesto, D., Williams, K., \& Fetherman, B. (2006). Reducing Foley catheter device days in an intensive care unit: Using the evidence to change practice. AACN Advanced Critical Care, 3, 272-283.

Saint, S. Clinical and economic consequences of nosocomial catheter-related bacteriuria. (2000). American Journal of Infection Control, 28, 68-75.

Saint, S., Lipcky, B.A., \& Goold, S. D. (2002). Indwelling Urinary Catheters: A One-Point Restraint? Annals of Internal Medicine, 137, 125-7. 
Schwartz, D. S., \& Barone, J. E. (2006). Correlation of urinalysis and dipstick results with catheter-associated urinary tract infections in surgical ICU patients. Intensive Care Medicine, 32, 1797-1801.

Stewart, P. S., \& Costerton, J. W. (2001). Antibiotic resistance of bacteria in biofilms. The Lancet, 358, 135-8.

Stickler, D. J. (2014). Clinical complications of urinary catheters caused by crystalline biofilms: Something needs to be done. Journal of Internal Medicine, 276, 120-9.

Tambyah, P. A., \& Maki, D. G. (2000). Catheter-associated urinary tract infection is rarely symptomatic: a prospective study of 1,497 catheterized patients. Archives of Internal Medicine, 160, 678-682.

Trautner, B. W., \& Darouiche, R. O. (2010). Role of biofilm in catheter-associated urinary tract infection. American Journal of Infection Control, 32, 177-83.

Tripepi-Bova, K. A., Sun, Z., Mason, D., \& Albert, N. M. (2013). Early removal of urinary catheters in patients with thoracic epidural catheters. Journal of Nursing Care Quality, 28, 340-344.

Umscheid, C. A., Mitchell, M. D., Doshi, J. A., Agarwal, R., Williams, K., \& Brennan, P. J.. (2011). Estimating the proportion of healthcare-associated infections that are reasonably preventable and the related mortality and costs. Infection Control and Hospital Epidemiology, 32, 101-114.

Verma, A., Bhani, D., Tomar, V., Bachhiwal, R., \& Yadav, S. (2016). Differences in Bacterial Colonization and Biofilm Formation Property of Uropathogens between the Two most Commonly used Indwelling Urinary Catheters. Journal of Clinical and Diagnostic Research, 10, PC01-PC03.

Weber, J., Purvis, S., VanDenBergh, S., \& Stevens, L. M. (2016). Standardizing Practice for Intermittent Irrigation of Indwelling Urinary Catheters. Journal of Nursing Care Quality, Epub.

Zimlichman, E., Henderson, D., Tamir, O., Franz, C., Song, P., Yamin, C. K., . . Bates, D. W.. (2013). Health care-associated infections: A meta-analysis of costs and financial impact on the US health care system. JAMA Internal Medicine, 173, 2039-2046. 


\section{Attachment A - Foley Insertion Checklist}

\begin{tabular}{|c|c|c|c|c|c|c|c|c|c|c|c|c|c|c|c|c|c|}
\hline \multicolumn{18}{|c|}{ Yes / No } \\
\hline $\begin{array}{c}\text { Completes } \\
\text { perineal care } \\
\text { with soap } \\
\text { and water } \\
\text { prior to } \\
\text { opening } \\
\text { catheter tray }\end{array}$ & $\begin{array}{c}\text { Performs } \\
\text { hand } \\
\text { hygiene prior } \\
\text { to procedure }\end{array}$ & $\begin{array}{c}\text { Opens the } \\
\text { tray making } \\
\text { a sterile field }\end{array}$ & $\begin{array}{l}\text { Places } \\
\text { underpad } \\
\text { shiny side } \\
\text { down }\end{array}$ & $\begin{array}{l}\text { Completes } \\
\text { pericare } \\
\text { using } \\
\text { provided } \\
\text { castile soap } \\
\text { wipes }\end{array}$ & $\begin{array}{c}\text { Removes } \\
\text { soiled gloves }\end{array}$ & $\begin{array}{c}\text { Completes } \\
\text { hand } \\
\text { hygiene with } \\
\text { provided } \\
\text { hand } \\
\text { sanitizer gel }\end{array}$ & $\begin{array}{c}\text { Dons sterile } \\
\text { gloves } \\
\text { maintaining } \\
\text { aseptic } \\
\text { technique }\end{array}$ & $\begin{array}{c}\text { Places } \\
\text { fenestrated } \\
\text { drape while } \\
\text { maintaining } \\
\text { aseptic } \\
\text { technique }\end{array}$ & $\begin{array}{c}\text { Dispenses } \\
\text { lubricant into } \\
\text { tray }\end{array}$ & $\begin{array}{c}\text { Removes top } \\
\text { tray and } \\
\text { places next } \\
\text { to bottom } \\
\text { tray (keeping } \\
\text { on CSR) }\end{array}$ & $\begin{array}{c}\text { Connects } \\
\text { sterile water } \\
\text { syringe to } \\
\text { inflation port }\end{array}$ & $\begin{array}{c}\text { Removes } \\
\text { Foley from } \\
\text { wrap }\end{array}$ & $\begin{array}{l}\text { Lubricates } \\
\text { Foley }\end{array}$ & $\begin{array}{c}\text { Cleanses } \\
\text { with PVI, } \\
\text { circle for } \\
\text { male, } \\
\text { downward } \\
\text { strokes for } \\
\text { female }\end{array}$ & $\begin{array}{c}\text { Insers Foley } \\
\text { aseptically }\end{array}$ & $\begin{array}{c}\text { Inflates } \\
\text { catheter } \\
\text { balloon with } \\
\text { entire } 10 \mathrm{~mL} \\
\text { of sterile } \\
\text { water }\end{array}$ & $\begin{array}{c}\text { Overall } \\
\text { aseptic } \\
\text { insertion }\end{array}$ \\
\hline 1 & 2 & 3 & 4 & 5 & 6 & 7 & 8 & 9 & 10 & 11 & 12 & 13 & 14 & 15 & 16 & 17 & 18 \\
\hline Yes / No & Yes / No & Yes / No & Yes / No & Yes / No & Yes / No & Yes / No & Yes / No & Yes / No & Yes / No & Yes / No & Yes / No & Yes / No & Yes / No & Yes / No & Yes / No & Yes / No & Yes / No \\
\hline
\end{tabular}




\section{Attachment B - Patient Refusal to Discontinue Foley Catheter Process}

\section{Discontinuation of Indwelling Urinary Catheters}

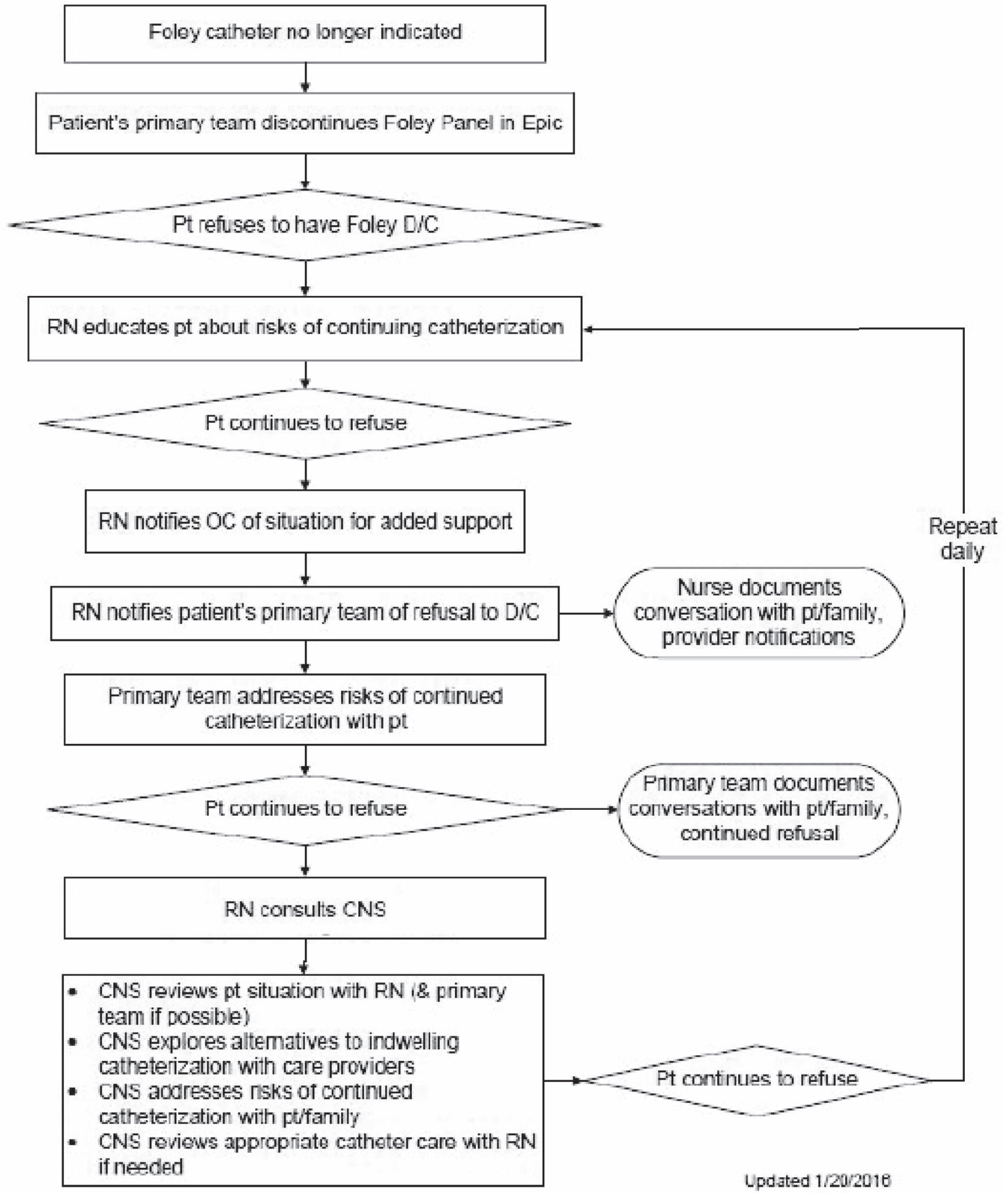




\section{Attachment C - Patient Admitted with Foley Catheter Process}

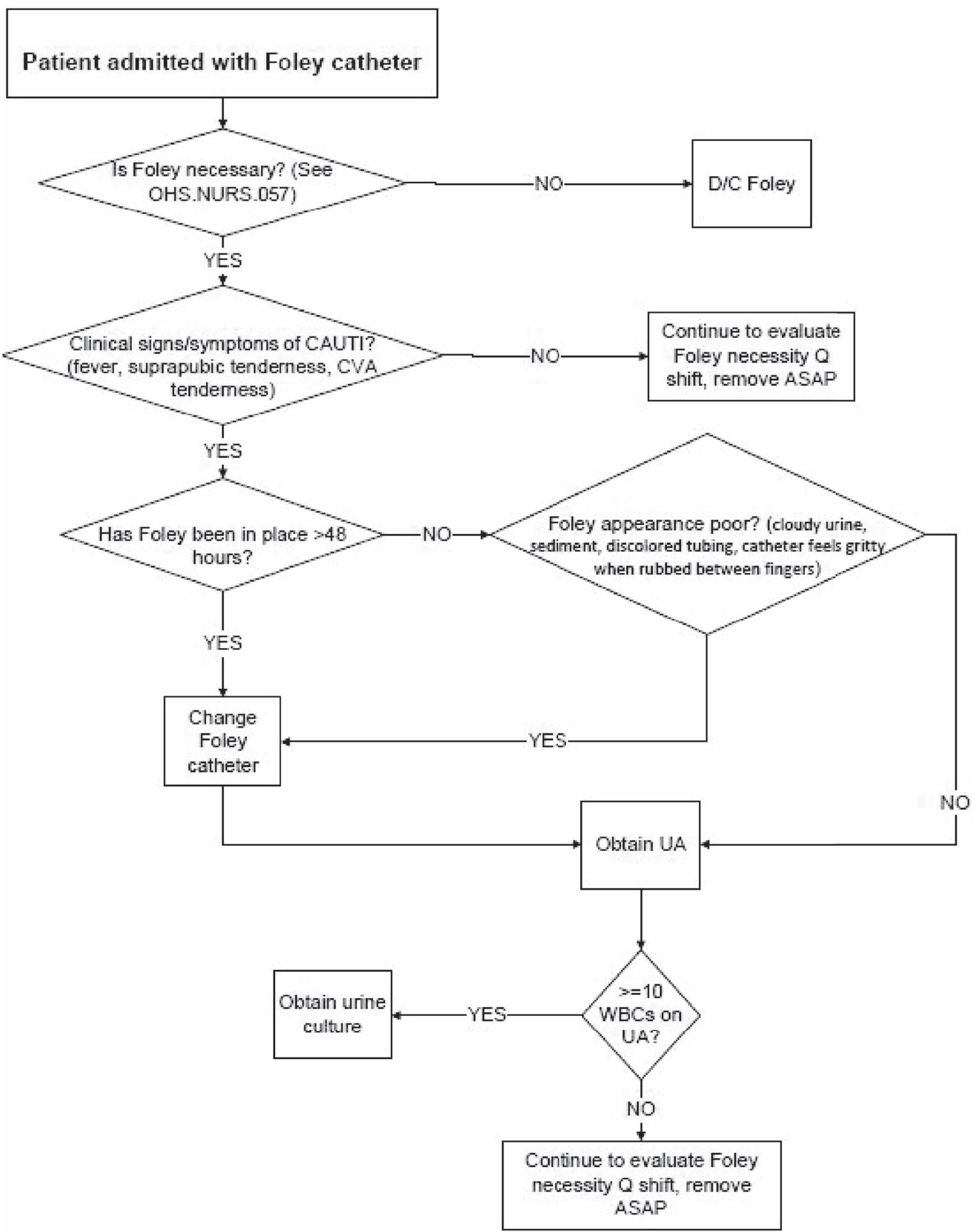




\section{Attachment D - Pediatric Foley Sizing}

\begin{tabular}{|l|l|l|}
\hline Age & Male Catheter Size & Female Catheter Size \\
\hline Premature infant & $3.5 \mathrm{Fr}$ to $5 \mathrm{Fr}$ & 3.5Fr to $5 \mathrm{Fr}$ \\
\hline Newborn & $5 \mathrm{Fr}$ & $5 \mathrm{Fr}$ to $6 \mathrm{Fr}$ \\
\hline Infant & $5 \mathrm{Fr}$ to $6 \mathrm{Fr}$ & $6 \mathrm{Fr}$ to $8 \mathrm{Fr}$ \\
\hline Toddler/preschool & $8 \mathrm{Fr}$ to $10 \mathrm{Fr}$ & 8Fr to $10 \mathrm{Fr}$ \\
\hline School-aged & 8Fr to $10 \mathrm{Fr}$ & 8Fr to $12 \mathrm{Fr}$ \\
\hline Adolescent & $12 \mathrm{Fr}$ & $12 \mathrm{Fr}$ to $14 \mathrm{Fr}$ \\
\hline
\end{tabular}

Lebet, R. (2008). Indwelling urinary catheter: Insertion and removal. In J.T. Verger \& R. M. Ledet (Eds.), AACN Procedure Manual for Pediatric Acute and Critical Care (pp 815-821). St. Louis, MO: Saunders Elsivier. 